\title{
TAK-264 (MLN0264) in Previously Treated Asian Patients with Advanced Gastrointestinal Carcinoma Expressing Guanylyl Cyclase C: Results from an Open-Label, Non-randomized Phase 1 Study
}

\author{
Yung-Jue Bang, MD, PhD ${ }^{1}$ \\ Toshimi Takano, MD² \\ Chia-Chi Lin, MD, PhD ${ }^{3}$ \\ Adedigbo Fasanmade, $\mathrm{PhD}^{4}$ \\ Huyuan Yang, $\mathrm{PhD}^{4}$ \\ Hadi Danaee, $\mathrm{SD}^{4}$ \\ Takayuki Asato, BSC ${ }^{4}$ \\ Thea Kalebic, MD, $\mathrm{PhD}^{4}$ \\ Hui Wang, $\mathrm{PhD}^{4}$ \\ Toshihiko Doi, MD, PhD
}

${ }^{1}$ Department of Internal Medicine, Seoul National University College of Medicine, Seoul, Korea, ${ }^{2}$ Department of Medical Oncology, Toranomon Hospital, Tokyo, Japan, ${ }^{3}$ Department of Oncology, National Taiwan University Hospital, Taipei, Taiwan, ${ }^{4}$ Millennium Pharmaceuticals, Inc., a wholly owned subsidiary of Takeda Pharmaceutical Company Limited, Cambridge, MA, USA, ${ }^{5}$ Department of Experimental Therapeutics, National Cancer Center Hospital East, Chiba, Japan

Correspondence: Yung-Jue Bang, MD, PhD Department of Internal Medicine, Seoul National University College of Medicine, Daehak-ro, Jongno-gu, Seoul 03080, Korea

Tel: $82-2-2072-2390$

Fax: 82-2-762-9662

E-mail: bangyj@snu.ac.kr

Received February 13, 2017

Accepted May 1, 2017

Published Online May 10, 2017

\begin{abstract}
Purpose
This phase 1 dose-escalation portion of the study evaluated the safety, pharmacokinetics (PK), and antitumor activity of TAK-264 in Asian patients with advanced gastrointestinal (GI) carcinoma or metastatic or recurrent gastric or gastroesophageal junction adenocarcinoma expressing guanylyl cyclase C (GCC).
\end{abstract}

\section{Materials and Methods}

Adult patients with advanced GI malignancies expressing GCC ( $\mathrm{H}$-score $\geq 10$ ) received TAK-264 on day 1 of 3-week cycles as 30-minute intravenous infusions for up to 1 year or until disease progression or unacceptable toxicity. The primary objectives were to evaluate the safety profile including dose-limiting toxicities (DLTs) during cycle 1, determine the maximum tolerated dose (MTD), and characterize the PK profile of TAK-264.

\section{Results}

Twelve patients were enrolled and treated with $1.2 \mathrm{mg} / \mathrm{kg}(\mathrm{n}=3), 1.5 \mathrm{mg} / \mathrm{kg}(\mathrm{n}=3)$, or $1.8 \mathrm{mg} / \mathrm{kg}$ TAK-264 ( $\mathrm{n}=6$ ). Median number of treatment cycles received was two (range, 1 to 10). None of the patients experienced a DLT and the MTD was not determined. Ten patients (83\%) experienced adverse events (AEs). The most common were neutropenia, anorexia, and nausea (each reported by four patients). Five patients (42\%) experienced grade $\geq 3$ AEs consisting of tumor hemorrhage and hypertension, ascites, adrenal insufficiency, neutropenia and asthenia. Serum exposure to TAK-264 increased proportionally with the dose and the median half-life was approximately 5.5-6.6 days. No patients experienced an objective response.

\section{Conclusion}

TAK-264 demonstrated a manageable safety profile with limited antitumor activity consistent with studies conducted in Western patients with advanced GI malignancies. TAK-264 exposure increased proportionally with the dose.

\section{Introduction}

Antibody-drug conjugates (ADCs) have revolutionized the field of cancer chemotherapy over the last two decades. ADCs use selective monoclonal antibodies that preferentially bind to the target tumor-associated antigens to deliver a highly potent cytotoxic payload [1]. ADCs, consisting of
Key words

Clinical trial, Gastrointestinal cancer, Stomach monoclonal antibodies conjugated to potent chemotherapeutics via a stable linker, have given rise to a highly efficacious class of anticancer drugs [2]. Currently, there are two ADCs approved, one for the treatment of relapsed/refractory Hodgkin's lymphoma and systemic anaplastic large cell lymphoma (brentuximab vedotin) [3], and the other for advanced/metastatic human epidermal growth factor receptor 2-positive breast cancer (trastuzumab emtansine) [4]. In 
addition, there is a rapidly growing clinical pipeline with over 60 ADCs in development to target a wide range of blood cancers and solid tumors [5].

Exploring the therapeutic potential of ADCs is of considerable interest in gastric cancer, which is recognized as a significant global health concern. On a global scale, each year there are approximately one million new cases and 750,000 deaths that account for $10 \%$ of all cancer-related deaths [6]. The highest incidence is found in Japan where gastric cancer is the second leading cause of death after lung cancer, with an estimated 110,000 new cases diagnosed and 54,000 deaths per year [7]. In China, there were approximately 400,000 new cases and 300,000 deaths from gastric cancer in 2005; while in Korea, there were approximately 28,000 new cases of gastric cancer in 2008 [8].

Globally, including in Asian countries, the combination of a fluorouracil (including oral fluoropyrimidines S-1 and capecitabine) and a platinum-containing compound (cisplatin or oxaliplatin) is the most widely accepted first-line chemotherapy for metastatic gastric cancer. However, many patients relapse and there are no standard treatment regimens in the second-line setting, although several phase 3 trials of second-line irinotecan, taxane, and ramucirumab have shown slight survival benefits [9]. In spite of current advances, clinical outcomes for patients with gastric carcinoma remain poor. Thus, there is a need to develop new treatment options.

The transmembrane cell surface receptor guanylyl cyclase $C$ (GCC) has been identified as a potential treatment target in patients with gastrointestinal (GI) cancers [10,11]. In normal tissues, GCC expression is restricted to the epithelial cells of the intestine, where it plays a key role in maintaining fluid ion homeostasis and genomic integrity $[12,13]$. Early findings suggest that GCC may play a role in tumorigenesis [12]. GCC expression has also been observed in tumors throughout the GI tract and their metastases: GCC is expressed in 60\%-70\% of gastric, pancreatic, and esophageal cancers [14-16] and $95 \%$ of primary and metastatic colorectal cancers $[10,17-20]$. In normal intestinal epithelial cells, access to GCC (expressed primarily to the luminal side) is restricted from the vascular compartment by the epithelial tight junctions [21]; however, in malignant cells, this anatomically privileged apical localization of GCC is disrupted, enabling agents targeting GCC to access tumor cells.

TAK-264 (formerly MLN0264) consists of a human monoclonal anti-GCC antibody conjugated to monomethyl auristatin $\mathrm{E}$ (MMAE) via a peptide linker [22]. The resulting ADC is internalized through receptor-mediated endocytosis into the target tumor cell and transported to the lysosomal compartment where enzymatic cleavage of the peptide linker releases MMAE, which in turn disrupts microtubule polymerization, leading to apoptotic cell death $[12,13]$. The tar- geted nature of ADCs allows for increased drug selectivity coupled with limited systemic exposure [23].

In the first-in-human study in patients (recruited in United States and Spain) with GI malignancies (NCT01577758), TAK-264 demonstrated a manageable safety profile at the maximum tolerated dose (MTD)/recommended phase 2 dose (RP2D) of $1.8 \mathrm{mg} / \mathrm{kg}$ every 21 days, and displayed early signals of clinical benefit in patients with pancreatic, esophageal, and gastric carcinomas [22]. Of the 39 responseevaluable patients, two patients with pancreatic carcinoma experienced a durable stable disease, while one patient with gastric adenocarcinoma achieved a partial response (PR).

The phase 1 dose-escalation portion of this open-label multi-center study was designed to evaluate the safety and tolerability of TAK-264 and to assess whether a MTD/RP2D of $1.8 \mathrm{mg} / \mathrm{kg}$ every 3 weeks or an alternative RP2D would be appropriate in Asian patients with advanced GI carcinoma.

\section{Materials and Methods}

\section{Patients}

Patients aged $\geq 18$ years diagnosed with GI carcinoma expressing GCC (H-score $\geq 10$, as indicated by immunohistochemistry [IHC]) were eligible [24]. Eligible GI malignancies included gastric carcinoma, esophageal carcinoma, colorectal carcinoma, small intestine carcinoma, pancreatic carcinoma, and biliary carcinoma. Patients were required to have an Eastern Cooperative Oncology Group performance status of 0 or 1 , adequate hematologic function as documented by absolute neutrophil count $(\mathrm{ANC}) \geq 1.5 \times 10^{9} / \mathrm{L}$, platelet count $\geq 100 \times 10^{9} / \mathrm{L}$, hemoglobin $\geq 9 \mathrm{~g} / \mathrm{dL}$, aspartate aminotransferase and alanine aminotransferase $\leq 2.5 \times$ upper limit of normal (ULN), albumin $\geq 3 \mathrm{~g} / \mathrm{dL}$, serum creatinine $\leq 1.5 \times$ ULN, and total bilirubin $\leq 1.5 \times$ ULN.

Patients with metastatic colorectal carcinoma or gastric carcinoma should have received $\geq 2$ prior anticancer therapies for advanced-stage disease and have completed chemotherapy, immunotherapy, or radiation therapy $\geq 4$ weeks prior to enrollment. Disease recurrence within 6 months of the last dose of post-surgical adjuvant chemotherapy accounted for one line of prior anticancer therapy for advanced disease.

Patients were excluded if they had radiotherapy within 3 weeks prior to enrollment, signs of grade $\geq 2$ peripheral neuropathy, concomitant cancer treatment, hormonal therapy, radiotherapy or immunotherapy, concurrent treatment within 4 weeks of study entry with another investigational drug, or treatment with any medication with clinical poten- 
tial risk to prolong the $\mathrm{QT}$ interval. Further exclusion criteria also included use of strong cytochrome P450 (CYP) 3A4 inhibitors within 2 weeks before the first dose of TAK-264 (in addition strong CYP3A4 inducers were not to be used in cycle 1 during the dose-limiting toxicity [DLT] evaluation period). Patients with ongoing anticoagulant therapy, history of myocardial infarction, unstable angina, or thromboembolic events within 6 months prior to enrollment, current uncontrolled cardiovascular conditions, symptomatic congestive heart failure were also excluded. Also not included, were patients with electrocardiogram (ECG) abnormalities considered by the investigator to be clinically significant, repeated baseline prolongation of the rate-corrected QT interval of electrocardiograph, history of another primary malignancy that had not been in remission for at least 3 years, with the exception of curatively treated nonmelanoma skin cancer or in situ carcinoma of the cervix uteri. Written informed consent was obtained from each patient prior to initiating study participation.

\section{Study design}

The study was conducted in compliance with the protocol, Good Clinical Practices, applicable regulatory requirements, and International Conference on Harmonisation guidelines. Institutional review boards and independent ethics committees at the participating investigational centers reviewed and approved all appropriate study documentation to safeguard the rights, safety, and well-being of the patients.

This open-label, non-randomized, multicenter study was conducted in Japan, Korea, and Taiwan in order to evaluate the effects of TAK-264 in Asian patients with GI malignancies. The study was designed to include a phase 1 dose-escalation portion to precede a phase 2 RP2D portion aimed at investigating efficacy and safety (ClinicalTrials.gov identifier: NCT02391038). The phase 1 portion of the study utilized a conventional $3+3$ dose-escalation scheme. Eligible patients received TAK-264 doses (planned dose levels, 1.2, 1.5, 1.8, $2.1,2.4$, and $2.7 \mathrm{mg} / \mathrm{kg}$ ) on day 1 of 3 -week cycles as 30-minute intravenous infusions for up to 1 year or until disease progression or unacceptable toxicity. At least one Japanese patient was included in evaluated dose groups. Neither a placebo nor an active control was included, and patient stratification was not planned.

Within the phase 2 portion of the study, an interim analysis for futility was planned to determine whether the results would support continuation of the trial and a completion of enrollment. The investigator-assessed complete response and PR rate was used as the endpoint for the interim analysis. The study was to be stopped on the basis of futility if there were fewer than two responses out of the 12 patients within each of the three groups with respective low, intermediate, and high GCC expression levels.

\section{Objectives and assessments}

The primary objectives for the phase 1 part of the study were (1) to evaluate the safety profile including DLTs of TAK-264, (2) to determine the RP2D, and (3) to characterize the pharmacokinetic (PK) profile of TAK-264, total antibody (TAb; conjugated and unconjugated), and MMAE. Secondary endpoints included the antitumor activity of TAK-264.

Archived tumor specimens were used for analysis using a validated, semi-quantitative GCC IHC assay to confirm the presence of GCC protein expression. GCC protein expression was characterized by an $\mathrm{H}$-score, a semi-quantitative scoring system that uses the sum of the percentage of tumor cells with a staining intensity of $1+, 2+$, and $3+$ [24]. Per the eligibility criteria, an $\mathrm{H}$-score of $\geq 10$ was confirmed prior to patient enrollment, implying that at least $10 \%$ of cells have shown GCC-positive staining.

DLTs were defined as any of the following events considered by the investigator to be at least possibly related to therapy with TAK-264 occurring within cycle 1 : grade 4 neutropenia (ANC $<500$ cells $/ \mathrm{mm}^{3}$ ); grade $\geq 3$ neutropenia with fever $\left(\geq 38.5^{\circ} \mathrm{C}\right)$ and / or infection; grade 4 thrombocytopenia (platelets $<25,000 / \mathrm{mm}^{3}$ ) or thrombocytopenia that requires platelet transfusion (with or without hemorrhage); grade $\geq 3$ thrombocytopenia with clinically meaningful bleeding at any time; anemia that requires blood transfusion; grade $\geq 3$ nausea and / or emesis that occurs despite the use of optimal antiemetic prophylaxis (includes both a 5-hydroxytryptamine 3 serotonin receptor antagonist and a corticosteroid given in standard doses and according to standard schedules); grade $\geq 3$ diarrhea that occurs despite optimal supportive care measures; any other grade $\geq 3$ non-hematologic toxicity (with the exception of fatigue lasting < 1 week); inability to start the next cycle of therapy due to treatment delay $>2$ weeks because of inadequate recovery from treatment-related toxicity (either hematologic or non-hematologic); or any other treatment-related grade $\geq 2$ non-hematologic toxicities that required TAK-264 dose reduction or discontinuation.

Thorough PK sampling to determine the serum concentrations of TAK-264 and TAb (conjugated and unconjugated) and the plasma concentrations of MMAE were performed in all patients. Blood specimens were taken pre-dose and postdose on day 1 , and on days $2,3,4,8$, and 15 of each cycle and at the end of treatment ( $30 \pm 7$ days after last dose).

Safety evaluations included adverse event (AE) evaluation (serious and non-serious), clinical laboratory assessments, and 12-lead ECGs. AEs were graded using the National Cancer Institute Common Terminology Criteria for Adverse Events ver. 4.03. Responses were evaluated using the modified Response Evaluation Criteria in Solid Tumors ver. 1.1. 
Table 1. Patient demographics and baseline characteristics

\begin{tabular}{lc} 
Characteristic & No. $(\%)(\mathbf{n}=\mathbf{1 2})$ \\
Age, median (range, yr) & $63(45-78)$ \\
Sex & \\
Male & $7(58)$ \\
Female & $5(42)$ \\
Race & \\
Korean & $7(58)$ \\
Japanese & $4(33)$ \\
Chinese & $1(8)$ \\
ECOG PS & \\
0 & $8(67)$ \\
1 & $4(33)$ \\
Cancer type & \\
Colon & $3(25)$ \\
Pancreatic & $3(25)$ \\
Rectal & $3(25)$ \\
Gastric & $3(25)$ \\
\hline
\end{tabular}

Values are presented as number (\%) unless otherwise indicated. ECOG PS, Eastern Cooperative Oncology Group performance status.

\section{Analysis populations and statistical analyses}

The safety population included all patients who received any dose of TAK-264. The response-evaluable population included all patients with measurable disease who received $\geq 1$ dose of TAK-264 and had $\geq 1$ post-baseline response assessment. The PK-evaluable population was defined as all patients who received $\geq 1$ dose of TAK-264 and who had suf- ficient TAK-264 concentration-time data to permit reliable estimation of TAK-264 exposure. The DLT-evaluable population was defined as all patients who either experienced DLT during cycle 1 or received their scheduled cycle 1 dose and completed all study procedures in cycle 1 without DLT.

Statistical analyses were planned to be primarily descriptive and graphical in nature with no formal statistical hypothesis.

\section{Results}

\section{Patient disposition and exposure}

Twelve patients with colon, pancreatic, rectal, and gastric cancers (three patients each) were enrolled in the phase 1 doseescalation portion of this study. Patient demographics and baseline characteristics are summarized in Table 1 . The majority of patients $(83 \%)$ had stage IV disease, and one patient had advanced pancreatic (T3NOM0) disease. All 12 patients received at least one prior anticancer therapy. Two of the 12 patients received prior radiation therapy. All patients had discontinued treatment due to disease progression.

Three patients received TAK-264 $1.2 \mathrm{mg} / \mathrm{kg}$, three patients received TAK-264 $1.5 \mathrm{mg} / \mathrm{kg}$, and six patients received TAK-264 $1.8 \mathrm{mg} / \mathrm{kg}$, on day 1 of 3-week cycles. Overall median number of study treatment cycles received was two (range, 1 to 10). Eight patients completed two cycles $(1.2 \mathrm{mg} / \mathrm{kg}, \mathrm{n}=3 ; 1.5 \mathrm{mg} / \mathrm{kg}, \mathrm{n}=2 ; 1.8 \mathrm{mg} / \mathrm{kg}, \mathrm{n}=3)$. Three patients completed more than two cycles (two patients treated with TAK-264 $1.8 \mathrm{mg} / \mathrm{kg}$ completed four and six cycles; one patient treated with TAK-264 $1.5 \mathrm{mg} / \mathrm{kg}$ completed 10 cycles).

Table 2. Most common (reported by $\geq 10 \%$ of patients) treatment-emergent adverse events by grade and severity

\begin{tabular}{lclll} 
& \multicolumn{4}{c}{ No. $(\%)(\mathbf{n}=\mathbf{1 2})$} \\
\cline { 2 - 5 } Adverse event & All grades & Grade $\mathbf{1}$ & Grade 2 & Grade $\mathbf{3 / 4}$ \\
Anorexia & $4(33.3)$ & $1(8.3)$ & $3(25.0)$ & 0 \\
Neutropenia & $4(33.3)$ & 0 & $3(25.0)$ & $1(8.3)$ \\
Nausea & $4(33.3)$ & $3(25.0)$ & $1(8.3)$ & 0 \\
Vomiting & $3(25.0)$ & $1(8.3)$ & $2(16.7)$ & 0 \\
Abdominal pain & $2(16.7)$ & 0 & $2(16.7)$ & 0 \\
Ascites & $2(16.7)$ & 0 & $1(8.3)$ & $1(8.3)$ \\
Constipation & $2(16.7)$ & 0 & $2(16.7)$ & 0 \\
Diarrhea & $2(16.7)$ & $1(8.3)$ & $1(8.3)$ & 0 \\
Fever & $2(16.7)$ & $2(16.7)$ & 0 & 0 \\
Asthenia & $2(16.7)$ & $1(8.3)$ & 0 & $1(8.3)$ \\
Myalgia & $2(16.7)$ & $2(16.7)$ & 0 & 0 \\
\hline
\end{tabular}


Table 3. Summary of PK parameters following cycle 1 administration of TAK-264 (PK-evaluable population)

\begin{tabular}{|c|c|c|c|}
\hline & $\begin{array}{c}\text { TAK-264 } \\
1.2 \mathrm{mg} / \mathrm{kg}(\mathrm{n}=3)\end{array}$ & $\begin{array}{c}\text { TAK-264 } \\
1.5 \mathrm{mg} / \mathrm{kg}(\mathrm{n}=3)\end{array}$ & $\begin{array}{c}\text { TAK-264 } \\
1.8 \mathrm{mg} / \mathrm{kg}(\mathrm{n}=6)\end{array}$ \\
\hline $\mathrm{C}_{\max }$, mean $(\mathrm{CV}, \mu \mathrm{g} / \mathrm{mL})$ & $25.62(3.5)$ & $33.84(18.3)$ & $35.77(20.2)$ \\
\hline $\mathrm{C}_{\text {trough, }}$ mean $(\mathrm{CV}, \mu \mathrm{g} / \mathrm{mL})$ & $0.20(5.63)^{a)}$ & $0.31(15.71)^{b)}$ & $0.26(30.45)$ \\
\hline $\mathrm{AUC}_{\text {inf, }}$ mean $(\mathrm{CV}$, day $\mu \mathrm{g} / \mathrm{mL})$ & $42.97(10.8)^{a)}$ & $63.58(10.1)^{\mathrm{b})}$ & $60.09(12.1)$ \\
\hline $\mathrm{T}_{\max }$, median (range, day) & $0.03(0.02-0.03)$ & $0.03(0.03-0.03)$ & $0.03(0.03-0.03)$ \\
\hline $\mathrm{T}_{1 / 2}$, median (range, day) & $5.49(5.44-5.53)^{\mathrm{a})}$ & $6.60(4.82-8.59)^{b)}$ & $5.46(4.70-6.54)$ \\
\hline
\end{tabular}

Values are presented as geometric mean (\% coefficient of variation [CV]) or median (range). PK, pharmacokinetic; $C_{\max }$ maximum observed concentration; $C_{\text {trough, }}$ plasma trough concentration measured at the end of a dosing interval; $\mathrm{AUC}_{\text {inf, }}$ area under the concentration-time curve from time 0 to infinity; $T_{\max }$, time to first occurrence of $C_{\max } ; T_{1 / 2}$, half-life. ${ }^{\text {a) }} n=2$, ${ }^{b} n=4$.

The majority of patients (75\%) received a cumulative total dose of TAK-264 of between $1.8 \mathrm{mg} / \mathrm{kg}$ and $3.6 \mathrm{mg} / \mathrm{kg}$.

\section{Safety}

None of the 12 patients experienced a DLT and the MTD was therefore not established. A grade 3 neutropenia event was observed in the $1.8 \mathrm{mg} / \mathrm{kg}$ dose cohort.

The safety profile of TAK-264 is summarized in Table 2 . Ten patients $(83 \%)$ experienced a treatment-emergent AE (TEAE) and five patients $(42 \%)$ experienced grade $\geq 3$ TEAEs, which consisted of tumor hemorrhage and hypertension, ascites, adrenal insufficiency, neutropenia, and asthenia. Five patients (42\%) experienced AEs that were assessed as related to study drug by the investigator and were primarily grade 1 and grade 2. Two patients experienced treatment-related grade $3 \mathrm{AEs}$; two instances of grade 3 neutropenia. Four patients experienced a serious AE that was considered unrelated to TAK-264. These were tumor hemorrhage and ascites; asthenia; abdominal distension; and adrenal insufficiency. These AEs were considered by the investigators disease related or resulted from disease progression.

Three patients required a dose reduction due to an $\mathrm{AE}$ (one with grade 2 neutropenia, two with grade 3 neutropenia) and one patient required a dose delay due to an $\mathrm{AE}$ (grade 2 neutropenia), all of which were considered related to TAK-264. One patient discontinued due to both disease progression and an $\mathrm{AE}$ (grade 3 tumor hemorrhage) which was considered by the investigator to be unrelated to TAK-264. No patients died while on study.

\section{Pharmacokinetics}

All 12 patients were included in the PK analysis (Table 3). Exposure to TAK-264, as measured by maximum observed concentration and area under the concentration-time curve, increased approximately proportionally with the dose. The median half-life (approximately 5.5-6.6 days) was similar for all dose cohorts (Table 3 ). The mean serum concentrations of TAK-264 and TAb and mean plasma concentration of MMAE were also generally dose dependent (data not shown).

\section{Antitumor activity}

All 12 patients were response evaluable. There were no objective responses; three patients had stable disease. One patient, a 48-year-old female diagnosed with T3N0M1 metastatic rectal cancer stage IV KRAS-mutant, who was treated with TAK-264 $1.5 \mathrm{mg} / \mathrm{kg}$ had stable disease $\geq 6$ months (duration, 225 days). Two additional patients, specifically a 63-year-old male affected by stage IV metastatic rectal cancer and a 56-year-old female with stage IV metastatic rectal cancer KRAS-mutant both treated with TAK-264 $1.8 \mathrm{mg} / \mathrm{kg}$ experienced a stable disease lasting respectively 81 and 46 days.

As the preliminary PK profile and overall response findings demonstrated similarities between the Western [22] and Asian patient populations, the study was terminated during the phase 1 portion and phase 2 was not initiated.

\section{Discussion}

TAK-264 administered as an intravenous infusion on day 1 of a 3-week cycle to Asian patients demonstrated a manageable safety profile in patients with recurrent or metastatic GI cancers. Common TEAEs reported in this study included anorexia, neutropenia, nausea, and vomiting, which were mostly grade 1 and 2 toxicities. Such a safety profile is consistent with that observed for TAK-264 in the Western population studies and for the ADCs brentuximab vedotin, polatuzumab vedotin, and pinatuzumab vedotin. These treatments were similarly associated with GI AEs 
(nausea and diarrhea), as well as fatigue, neutropenia, diarrhea, pyrexia, and peripheral neuropathy, in patients with hematologic malignancies [25-27]. During this study, one patient $(8 \%)$ experienced grade 1 peripheral neuropathy, which is consistent with the prior clinical experiences of GCC-positive patients with GI malignancies [22] who participated in earlier phase 1 and phase 2 studies of TAK-264 [28].

The limited activity observed in this Asian population was consistent with that observed in other studies of TAK-264 in comparable Western patient populations who participated in phase 1 [22] and phase 2 [28] studies, where objective response rates (all PRs) of 3\% and 6\%, respectively, have been shown. In all three studies, increases in exposure to TAK-264 and free MMAE were approximately proportional to dose observed. Detectable serum concentrations of ADCs were evident prior to the commencement of cycle 2 (shown in Table 3 as plasma trough concentration measured at the end of a dosing interval), which suggests that the limited antitumor activity observed in those patients was not a consequence of a lack of circulating levels of the ADC (TAK-264) in the blood. The systemic exposure to TAK-264 observed in this study is similar to those reported for the Western population [22]. Due to compelling similarities between the Western and Asian patient populations, further clinical investigation in Asian patients was not considered critical to estimating the therapeutic potential of this experimental agent. The study was terminated and the phase 2 portion was not initiated.

In most of the ADCs in development to date, the cytotoxic payload and lysine or cysteine residues on the targeting antibody are non-selectively conjugated (via a stable or cleavable linker) [29,30]. This may result in a variable drug-antibody ratio and a heterogeneous ADC product, potentially impacting on clinical efficacy and safety $[29,30]$. It is expected that site-specific conjugation can maintain antitumor efficacy while reducing systemic toxicity, improving the therapeutic index of an ADC [31]. Alternative payloads also have the potential to improve the clinical efficacy of the next generation of ADCs [29]. Given the high frequency of its expression in GI cancers, GCC remains a promising target for future ADC development.

In conclusion, the clinical experience with TAK-264 in Asian patients is comparable to that observed in Western patients, with a manageable safety profile and limited antitumor activity. Given the modest clinical benefit observed in this and other trials of TAK-264 in patients with advanced GI malignancies, further evaluation of single-agent TAK-264 is not warranted in this setting. Further development of ADCs targeting GCC may lead to more effective therapeutic modalities.

\section{Conflicts of Interest}

Yung-Jue Bang, Chia-Chi Lin, Toshimi Takano, and Toshihiko Doi disclose no conflicts of interest. Adedigbo Fasanmade, Huyuan Yang, Hadi Danaee, Takayuki Asato, Thea Kalebic, and Hui Wang are employees of Takeda Pharmaceuticals. Takayuki Asato holds stocks or shares in Takeda Pharmaceutical Company Limited and Chugai Pharmaceutical Company Limited. Thea Kalebic holds stocks in Takeda Pharmaceutical Company Limited.

This study was funded by Millennium Pharmaceuticals Inc., a wholly owned subsidiary of Takeda Pharmaceutical Company Limited.

\section{Acknowledgments}

The authors acknowledge Sabah Farooq and Victoria A. Robb of FireKite, an Ashfield company, part of UDG Healthcare plc, for writing support during the development of this manuscript, which was funded by Millennium Pharmaceuticals, Inc., ADC technology was licensed from Seattle Genetics, Inc., Washington, USA.

\section{References}

1. Teicher BA, Chari RV. Antibody conjugate therapeutics: challenges and potential. Clin Cancer Res. 2011;17:6389-97.

2. Panowski S, Bhakta S, Raab H, Polakis P, Junutula JR. Site-specific antibody drug conjugates for cancer therapy. MAbs. 2014;6:34-45.

3. Chen R, Gopal AK, Smith SE, Ansell SM, Rosenblatt JD, Savage KJ, et al. Five-year survival and durability results of brentuximab vedotin in patients with relapsed or refractory Hodgkin lymphoma. Blood. 2016;128:1562-6.

4. Van den Mooter T, Teuwen LA, Rutten A, Dirix L. Trastu- zumab emtansine in advanced human epidermal growth factor receptor 2-positive breast cancer. Expert Opin Biol Ther. 2015;15:749-60.

5. Tsuchikama K, An Z. Antibody-drug conjugates: recent advances in conjugation and linker chemistries. Protein Cell. 2016 Oct 14 [Epub]. https://doi.org/10.1007/s13238-0160323-0.

6. Jemal A, Bray F, Center MM, Ferlay J, Ward E, Forman D. Global cancer statistics. CA Cancer J Clin. 2011;61:69-90.

7. Koizumi W. Chemotherapy for advanced gastric cancer: 
review of global and Japanese status. Gastrointest Cancer Res. 2007;1:197-203.

8. Shin A, Kim J, Park S. Gastric cancer epidemiology in Korea. J Gastric Cancer. 2011;11:135-40.

9. Kanagavel D, Fedyanin M, Tryakin A, Tjulandin S. Secondline treatment of metastatic gastric cancer: Current options and future directions. World J Gastroenterol. 2015;21:1162135.

10. Carrithers SL, Parkinson SJ, Goldstein SD, Park PK, Urbanski RW, Waldman SA. Escherichia coli heat-stable enterotoxin receptors: a novel marker for colorectal tumors. Dis Colon Rectum. 1996;39:171-81.

11. Hyslop T, Waldman SA. Guanylyl cyclase C as a biomarker in colorectal cancer. Biomark Med. 2013;7:159-67.

12. Li P, Schulz S, Bombonati A, Palazzo JP, Hyslop TM, Xu Y, et al. Guanylyl cyclase $C$ suppresses intestinal tumorigenesis by restricting proliferation and maintaining genomic integrity. Gastroenterology. 2007;133:599-607.

13. Vaandrager AB. Structure and function of the heat-stable enterotoxin receptor/guanylyl cyclase C. Mol Cell Biochem. 2002;230:73-83.

14. John M, Wiedenmann B, Kruhoffer M, Adermann K, Ankorina-Stark I, Schlatter E, et al. Guanylin stimulates regulated secretion from human neuroendocrine pancreatic cells. Gastroenterology. 1998;114:791-7.

15. Kloeters O, Friess H, Giese N, Buechler MW, Cetin Y, Kulaksiz $\mathrm{H}$. Uroguanylin inhibits proliferation of pancreatic cancer cells. Scand J Gastroenterol. 2008;43:447-55.

16. Park J, Schulz S, Haaf J, Kairys JC, Waldman SA. Ectopic expression of guanylyl cyclase $\mathrm{C}$ in adenocarcinomas of the esophagus and stomach. Cancer Epidemiol Biomarkers Prev. 2002;11:739-44.

17. Carrithers SL, Parkinson SJ, Goldstein S, Park P, Robertson DC, Waldman SA. Escherichia coli heat-stable toxin receptors in human colonic tumors. Gastroenterology. 1994;107:1653-61.

18. Buc E, Vartanian MD, Darcha C, Dechelotte P, Pezet D. Guanylyl cyclase $\mathrm{C}$ as a reliable immunohistochemical marker and its ligand Escherichia coli heat-stable enterotoxin as a potential protein-delivering vehicle for colorectal cancer cells. Eur J Cancer. 2005;41:1618-27.

19. Camci C, Sahin A, Sevinc A, Kalender ME, Oztuzcu S, Sever ON, et al. Peripheral blood guanylyl cyclase c (GCC) expressions are associated with prognostic parameters and response to therapy in colorectal cancer patients. Tumour Biol. 2011;32: 1265-70.

20. Winn B, Tavares R, Matoso A, Noble L, Fanion J, Waldman $\mathrm{SA}$, et al. Expression of the intestinal biomarkers Guanylyl cyclase $\mathrm{C}$ and $\mathrm{CDX} 2$ in poorly differentiated colorectal carci- nomas. Hum Pathol. 2010;41:123-8.

21. Wolfe HR, Mendizabal M, Lleong E, Cuthbertson A, Desai V, Pullan S, et al. In vivo imaging of human colon cancer xenografts in immunodeficient mice using a guanylyl cyclase C-specific ligand. J Nucl Med. 2002;43:392-9.

22. Almhanna K, Kalebic T, Cruz C, Faris JE, Ryan DP, Jung J, et al. Phase I study of the investigational anti-guanylyl cyclase antibody-drug conjugate TAK-264 (MLN0264) in adult patients with advanced gastrointestinal malignancies. Clin Cancer Res. 2016;22:5049-57.

23. Peters C, Brown S. Antibody-drug conjugates as novel anticancer chemotherapeutics. Biosci Rep. 2015;35:e00225.

24. van Diest PJ, van Dam P, Henzen-Logmans SC, Berns E, van der Burg ME, Green J, et al. A scoring system for immunohistochemical staining: consensus report of the task force for basic research of the EORTC-GCCG. European Organization for Research and Treatment of Cancer-Gynaecological Cancer Cooperative Group. J Clin Pathol. 1997;50:801-4.

25. Younes A, Gopal AK, Smith SE, Ansell SM, Rosenblatt JD, Savage KJ, et al. Results of a pivotal phase II study of brentuximab vedotin for patients with relapsed or refractory Hodgkin's lymphoma. J Clin Oncol. 2012;30:2183-9.

26. Pro B, Advani R, Brice P, Bartlett NL, Rosenblatt JD, Illidge T, et al. Brentuximab vedotin (SGN-35) in patients with relapsed or refractory systemic anaplastic large-cell lymphoma: results of a phase II study. J Clin Oncol. 2012;30:2190-6.

27. Morschhauser F, Flinn I, Advani RH, Diefenbach CS, Kolibaba $\mathrm{K}$, Press OW, et al. Updated results of a phase II randomized study (ROMULUS) of polatuzumab vedotin or pinatuzumab vedotin plus rituximab in patients with relapsed/refractory non-Hodgkin lymphoma. Blood. 2014;124:4457.

28. Almhanna K, Miron M, Wright D, Gracian A, Hubner R, Van Laethem J, et al. Phase 2 trial of TAK-264 (MLN0264) in previously-treated patients with metastatic or recurrent adenocarcinoma of the stomach or gastroesophageal junction expressing guanylyl cyclase C. In: AACR Annual Meeting 2016; 2016 Apr 16-20; New Orleans, LA, USA. Philadelphia, PA: American Association for Cancer Research; 2016. Abstr CT107.

29. Diamantis N, Banerji U. Antibody-drug conjugates: an emerging class of cancer treatment. Br J Cancer. 2016;114:362-7.

30. Garcia-Echeverria C. Developing second generation antibodydrug conjugates: the quest for new technologies. J Med Chem. 2014;57:7888-9.

31. Junutula JR, Raab H, Clark S, Bhakta S, Leipold DD, Weir S, et al. Site-specific conjugation of a cytotoxic drug to an antibody improves the therapeutic index. Nat Biotechnol. 2008;26:925-32. 